\title{
FINDING THE LARGEST EIGENVALUE OF A NONNEGATIVE TENSOR $^{*}$
}

\author{
MICHAEL NG ${ }^{\dagger}$, LIQUN QI ${ }^{\ddagger}$, AND GUANGLU ZHOU§ \\ In memory of Gene Golub
}

\begin{abstract}
In this paper we propose an iterative method for calculating the largest eigenvalue of an irreducible nonnegative tensor. This method is an extension of a method of Collatz (1942) for calculating the spectral radius of an irreducible nonnegative matrix. Numerical results show that our proposed method is promising. We also apply the method to studying higher-order Markov chains.
\end{abstract}

Key words. spectral radius, nonnegative tensor, iterative method, higher-order Markov chains

AMS subject classifications. 15A18, 15A69, 65F15

DOI. $10.1137 / 09074838 \mathrm{X}$

1. Introduction. Let $R$ be the real field. We consider an $m$-order $n$-dimensional tensor $\mathcal{A}$ consisting of $n^{m}$ entries in $R$ :

$$
\mathcal{A}=\left(A_{i_{1} i_{2} \ldots i_{m}}\right), \quad A_{i_{1} i_{2} \ldots i_{m}} \in R, \quad 1 \leq i_{1}, i_{2}, \ldots, i_{m} \leq n .
$$

$\mathcal{A}$ is called nonnegative (or, respectively, positive) if $A_{i_{1} i_{2} \ldots i_{m}} \geq 0$ (or, respectively, $\left.A_{i_{1} i_{2} \ldots i_{m}}>0\right)$. To an $n$-dimensional column vector $x=\left[x_{1}, x_{2}, \ldots, x_{n}\right]^{T} \in R^{n}$, real or complex, we define an $n$-dimensional column vector:

$$
\mathcal{A} x^{m-1}:=\left(\sum_{i_{2}, \ldots, i_{m}=1}^{n} A_{i i_{2} \ldots i_{m}} x_{i_{2}} \cdots x_{i_{m}}\right)_{1 \leq i \leq n} .
$$

Definition 1.1. Let $\mathcal{A}$ be an m-order n-dimensional tensor and $C$ be the set of all complex numbers. Assume that $\mathcal{A} x^{m-1}$ is not identical to zero. We say that $(\lambda, x) \in C \times\left(C^{n} \backslash\{0\}\right)$ is an eigenvalue-eigenvector of $\mathcal{A}$ if

$$
\mathcal{A} x^{m-1}=\lambda x^{[m-1]} .
$$

Here, $x^{[\alpha]}=\left[x_{1}^{\alpha}, x_{2}^{\alpha}, \ldots, x_{n}^{\alpha}\right]^{T}$.

This definition was introduced by Qi [9] when $m$ is even and $\mathcal{A}$ is symmetric. Independently, Lim [7] gave such a definition but restricted $x$ to be a real vector and $\lambda$ to be a real number. Lim also discussed the case that $m$ is odd, by using $\left[x_{1}^{m-1} \operatorname{sign}\left(x_{1}\right), x_{2}^{m-1} \operatorname{sign}\left(x_{2}\right), \ldots, x_{n}^{m-1} \operatorname{sign}\left(x_{n}\right)\right]^{T}$ on the right-hand side instead. Here we use the definition given by Chang, Pearson, and Zhang [2]. This definition is slightly different from the definition of Lim [7] when $m$ is odd.

${ }^{*}$ Received by the editors February 2, 2009; accepted for publication (in revised form) by L. De Lathauwer June 9, 2009; published electronically August 28, 2009.

http://www.siam.org/journals/simax/31-3/74838.html

$\dagger$ Department of Mathematics, The Hong Kong Baptist University, Hong Kong (mng@math.hkbu. edu.hk). This author's research was supported in part by RGC grants and HKBU FRGs.

${ }^{\ddagger}$ Department of Applied Mathematics, The Hong Kong Polytechnic University, Hong Kong (maqilq@polyu.edu.hk). This author's work was supported by the Hong Kong Research Grant Council.

$\S$ Department of Mathematics and Statistics, Curtin University of Technology, Perth, WA 6102, Australia(G.Zhou@curtin.edu.au).

1090 
Eigenvalues of higher order have become an important topic of study in a new applied mathematics branch, numerical multilinear algebra, and they have a wide range of practical applications; for more references, see $[7,10,11,12]$. In recent studies of numerical multilinear algebra $[2,3,7,9,10]$, eigenvalue problems for tensors have attracted special attention. In particular, in $[2,7]$ the Perron-Frobenius theorem for nonnegative matrices has been generalized to the class of nonnegative tensors.

Definition 1.2 (see [2]). An m-order $n$-dimensional tensor $\mathcal{A}$ is called reducible if there exists a nonempty proper index subset $I \subset\{1,2, \ldots, n\}$ such that

$$
A_{i_{1} i_{2} \ldots i_{m}}=0 \quad \forall i_{1} \in I, \forall i_{2}, \ldots, i_{m} \notin I .
$$

If $\mathcal{A}$ is not reducible, then we call $\mathcal{A}$ irreducible.

The Perron-Frobenius theorem for nonnegative tensors is related to measuring higher-order connectivity in linked objects and hypergraphs [7]. In [2] the well-known Collatz [6] minimax theorem for irreducible nonnegative matrices has been extended to irreducible nonnegative tensors. In the following, we state the Perron-Frobenius theorem for nonnegative tensors.

TheOREM 1.3 (see [2]). If $\mathcal{A}$ is an irreducible nonnegative tensor of order $m$ and dimension $n$, then there exist $\lambda_{0}>0$ and $x_{0}>0, x_{0} \in R^{n}$, such that

$$
\mathcal{A} x_{0}^{m-1}=\lambda_{0} x_{0}^{[m-1]} .
$$

Moreover, if $\lambda$ is an eigenvalue with a nonnegative eigenvector, then $\lambda=\lambda_{0}$. If $\lambda$ is an eigenvalue of $\mathcal{A}$, then $|\lambda| \leq \lambda_{0}$.

Let $P=\left\{x \in R^{n}: x_{i} \geq 0,1 \leq i \leq n\right\}$ and $\operatorname{int}(P)=\left\{x \in R^{n}: x_{i}>0,1 \leq i \leq\right.$ $n\}$. We have the following minimax theorem for irreducible nonnegative tensors. We will give the definition of an irreducible nonnegative tensor at the beginning of the next section.

Theorem 1.4 (see [2]). Assume that $\mathcal{A}$ is an irreducible nonnegative tensor of order $m$ and dimension $n$. Then

$$
\min _{x \in \operatorname{int}(P)} \max _{x_{i}>0} \frac{\left(\mathcal{A} x^{m-1}\right)_{i}}{x_{i}^{m-1}}=\lambda_{0}=\max _{x \in \operatorname{int}(P)} \min _{x_{i}>0} \frac{\left(\mathcal{A} x^{m-1}\right)_{i}}{x_{i}^{m-1}},
$$

where $\lambda_{0}$ is the unique positive eigenvalue corresponding to the positive eigenvector.

Based on the above results, in section 2 we propose an iterative method for computing the largest eigenvalue of an irreducible nonnegative tensor. This method can be regarded as an extension of a method of Collatz [6] (see [13, 14]) for calculating the spectral radius of an irreducible nonnegative matrix. In section 3 we report our numerical results, which show that our proposed method is promising. In section 4 , we apply the method to studying higher-order Markov chains. We conclude the paper with some remarks in section 5 .

2. An iterative method. In this section, we give an iterative method for calculating the largest eigenvalue of an irreducible nonnegative tensor. We first give some results which will be used later.

Lemma 2.1 (see [2]). If an m-order $n$-dimensional nonnegative tensor $\mathcal{A}$ is irreducible, then

$$
\sum_{i_{2}, \ldots, i_{m}=1}^{n} A_{i i_{2} \ldots i_{m}}>0 \quad \forall 1 \leq i \leq n
$$

Copyright $@$ by SIAM. Unauthorized reproduction of this article is prohibited. 
From this lemma, we have the following result.

Lemma 2.2. If an m-order $n$-dimensional nonnegative tensor $\mathcal{A}$ is irreducible, then for any positive vector $x>0, x \in R^{n}, \mathcal{A} x^{m-1}$ is a positive vector; i.e.,

$$
\mathcal{A} x^{m-1}>0 \text {. }
$$

By a direct computation, we obtain the following lemma.

Lemma 2.3. Suppose that $\mathcal{A}$ is a nonnegative tensor of order $m$ and dimension $n, x$ and $y$ are two nonnegative column vectors, and $t$ is a positive number. Then, we have the following:

(1) If $x \geq y$, then $\mathcal{A} x^{m-1} \geq \mathcal{A} y^{m-1}$;

(2) $\mathcal{A}(t x)^{m-1}=t^{m-1} \mathcal{A} x^{m-1}$.

Now we give the main result of this paper. Based on this result, we will obtain an iterative method for calculating a lower bound and an upper bound of the largest eigenvalue of an irreducible nonnegative tensor.

THeOREM 2.4. Let $\mathcal{A}$ be an irreducible nonnegative tensor of order $m$ and dimension $n$ and let $x^{(0)} \in R^{n}$ be an arbitrary positive vector. Let $y^{(0)}=\mathcal{A}\left(x^{(0)}\right)^{m-1}$. Define

$$
\begin{aligned}
& x^{(1)}=\frac{\left(y^{(0)}\right)^{\left[\frac{1}{m-1}\right]}}{\|\left(y^{(0)}\right)^{\left[\frac{1}{m-1}\right]},}, \quad y^{(1)}=\mathcal{A}\left(x^{(1)}\right)^{m-1}, \\
& x^{(2)}=\frac{\left(y^{(1)}\right)^{\left[\frac{1}{m-1}\right]}}{\|\left(y^{(1)}\right)^{\left[\frac{1}{m-1}\right]},}, \quad y^{(2)}=\mathcal{A}\left(x^{(2)}\right)^{m-1}, \\
& \vdots \\
& x^{(k+1)}=\frac{\left(y^{(k)}\right)^{\left[\frac{1}{m-1}\right]}}{\left\|\left(y^{(k)}\right)^{\left[\frac{1}{m-1}\right]}\right\|}, \quad y^{(k+1)}=\mathcal{A}\left(x^{(k+1)}\right)^{m-1}, \quad k \geq 2,
\end{aligned}
$$

and let

$$
\underline{\lambda}_{k}=\min _{x_{i}^{(k)}>0} \frac{\left(\mathcal{A}\left(x^{(k)}\right)^{m-1}\right)_{i}}{\left(x_{i}^{(k)}\right)^{m-1}}, \quad \bar{\lambda}_{k}=\max _{x_{i}^{(k)}>0} \frac{\left(\mathcal{A}\left(x^{(k)}\right)^{m-1}\right)_{i}}{\left(x_{i}^{(k)}\right)^{m-1}}, \quad k=1,2, \ldots
$$

Assume that $\lambda_{0}$ is the unique positive eigenvalue corresponding to a nonnegative eigenvector. Then,

$$
\underline{\lambda}_{1} \leq \underline{\lambda}_{2} \leq \cdots \leq \lambda_{0} \leq \cdots \leq \bar{\lambda}_{2} \leq \bar{\lambda}_{1}
$$

Proof. Clearly, by Theorem 1.4, for $k=1,2, \ldots$,

$$
\underline{\lambda}_{k} \leq \lambda_{0} \leq \bar{\lambda}_{k}
$$

We now prove for any $k \geq 1$

$$
\underline{\lambda}_{k} \leq \underline{\lambda}_{k+1} \quad \text { and } \quad \bar{\lambda}_{k+1} \leq \bar{\lambda}_{k}
$$

Copyright (c) by SIAM. Unauthorized reproduction of this article is prohibited. 
For each $k=1,2, \ldots$, by the definition of $\underline{\lambda}_{k}$, we have

$$
\mathcal{A}\left(x^{(k)}\right)^{m-1} \geq \underline{\lambda}_{k}\left(x^{(k)}\right)^{[m-1]}>0 .
$$

Then,

$$
\left(y^{(k)}\right)^{\left[\frac{1}{m-1}\right]}=\left\{\mathcal{A}\left(x^{(k)}\right)^{m-1}\right\}^{\left[\frac{1}{m-1}\right]} \geq\left(\underline{\lambda}_{k}\right)^{\frac{1}{m-1}} x^{(k)}>0 .
$$

So,

$$
x^{(k+1)}=\frac{\left(y^{(k)}\right)^{\left[\frac{1}{m-1}\right]}}{\left\|\left(y^{(k)}\right)^{\left[\frac{1}{m-1}\right]}\right\|} \geq \frac{\left(\underline{\lambda}_{k}\right)^{\frac{1}{m-1}} x^{(k)}}{\left\|\left(y^{(k)}\right)^{\left[\frac{1}{m-1}\right]}\right\|}>0 .
$$

Hence, by Lemma 2.3, we get

$$
\begin{aligned}
\mathcal{A}\left(x^{(k+1)}\right)^{m-1} & \geq \frac{\underline{\lambda}_{k} \mathcal{A}\left(x^{(k)}\right)^{m-1}}{\left\|\left(y^{(k)}\right)^{\left[\frac{1}{m-1}\right]}\right\|^{m-1}}=\frac{\underline{\lambda}_{k} y^{(k)}}{\left\|\left(y^{(k)}\right)^{\left[\frac{1}{m-1}\right]}\right\|^{m-1}}=\frac{\underline{\lambda}_{k}\left\{\left(y^{(k)}\right)^{\left[\frac{1}{m-1}\right]}\right\}^{[m-1]}}{\left\|\left(y^{(k)}\right)^{\left[\frac{1}{m-1}\right]}\right\|^{m-1}}, \\
& =\underline{\lambda}_{k}\left(x^{(k+1)}\right)^{[m-1]},
\end{aligned}
$$

which means, for each $i=1,2, \ldots, n$,

$$
\underline{\lambda}_{k} \leq \frac{\left(\mathcal{A}\left(x^{(k+1)}\right)^{m-1}\right)_{i}}{\left(x_{i}^{(k+1)}\right)^{m-1}} .
$$

Therefore, we obtain

$$
\underline{\lambda}_{k} \leq \underline{\lambda}_{k+1}
$$

Similarly, we can prove that

$$
\bar{\lambda}_{k+1} \leq \bar{\lambda}_{k}
$$

This completes our proof.

Based on Theorem 2.4, we state our algorithm as follows.

Algorithm 2.1.

Step 0. Choose $x^{(0)}>0, x^{(0)} \in R^{n}$. Let $y^{(0)}=\mathcal{A}\left(x^{(0)}\right)^{m-1}$ and set $k:=0$.

Step 1. Compute

$$
\begin{aligned}
x^{(k+1)} & =\frac{\left(y^{(k)}\right)^{\left[\frac{1}{m-1}\right]}}{\|\left(y^{(k)}\right)^{\left[\frac{1}{m-1}\right]},}, \\
y^{(k+1)} & =\mathcal{A}\left(x^{(k+1)}\right)^{m-1}, \\
\underline{\lambda}_{k+1} & =\min _{x_{i}^{(k+1)}>0} \frac{\left(y^{(k+1)}\right)_{i}}{\left(x_{i}^{(k+1)}\right)^{m-1}}, \\
\bar{\lambda}_{k+1} & =\max _{x_{i}^{(k+1)}>0} \frac{\left(y^{(k+1)}\right)_{i}}{\left(x_{i}^{(k+1)}\right)^{m-1}} .
\end{aligned}
$$

Copyright $@$ by SIAM. Unauthorized reproduction of this article is prohibited. 
Step 2. If $\bar{\lambda}_{k+1}=\underline{\lambda}_{k+1}$, stop. Otherwise, replace $k$ by $k+1$ and go to Step 1 .

For Algorithm 2.1, by Theorem 2.4, we obtain the following result.

TheOREM 2.5. Let $\mathcal{A}$ be an irreducible nonnegative tensor of order $m$ and dimension n. Assume that $\lambda_{0}$ is the unique positive eigenvalue corresponding to a nonnegative eigenvector. Then, Algorithm 2.1 produces the value of $\lambda_{0}$ in a finite number of steps or generates two convergent sequences $\left\{\underline{\lambda}_{k}\right\}$ and $\left\{\bar{\lambda}_{k}\right\}$. Furthermore, let $\underline{\lambda}=\lim _{k \rightarrow+\infty} \underline{\lambda}_{k}$ and $\bar{\lambda}=\lim _{k \rightarrow+\infty} \bar{\lambda}_{k}$. Then, $\underline{\lambda}$ and $\bar{\lambda}$ are a lower bound and an upper bound, respectively, of $\lambda_{0}$. If $\underline{\lambda}=\bar{\lambda}$, then $\lambda_{0}=\underline{\lambda}=\bar{\lambda}$.

Since $\left\{\underline{\lambda}_{k}\right\}$ is a monotonic increasing sequence and has an upper bound, the limit exists. This implies that $\left\{x^{(k)}\right\}$ converges to a vector $x$.

3. Numerical results. In this section, in order to show the viability of Algorithm 2.1, we used MATLAB 7.1 to test it on Example 1 and some randomly generated tensors. For these randomly generated tensors, the value of each entry is between 0 and 10. Throughout the computational experiments, $x^{(0)}=[1,1, \ldots, 1]^{T} \in R^{n}$. We terminate our iteration when one of the following conditions is satisfied:

(1) $k \geq 100$,

(2) $\bar{\lambda}_{k}-\underline{\lambda}_{k} \leq 10^{-7}$.

Example 1. Consider the 3-order 3-dimensional tensor

$$
\mathcal{A}=[A(1,:,:), A(2,:,:), A(3,:,:)]
$$

where

$$
\begin{aligned}
& A(1,:,:)=\left(\begin{array}{lll}
6.48 & 8.35 & 1.03 \\
4.04 & 3.72 & 1.43 \\
6.61 & 6.41 & 1.35
\end{array}\right), \\
& A(2,:,:)=\left(\begin{array}{lll}
9.02 & 0.78 & 6.90 \\
9.70 & 4.79 & 1.85 \\
2.09 & 4.17 & 2.98
\end{array}\right), \\
& A(3,:,:)=\left(\begin{array}{lll}
9.55 & 1.57 & 6.89 \\
5.63 & 5.55 & 1.45 \\
5.65 & 8.29 & 6.22
\end{array}\right) .
\end{aligned}
$$

Our numerical results are reported in Tables 1 and 2. In Table 1, we give the output of Algorithm 2.1 for the 3-order 3-dimensional tensor of Example 1, where $\lambda_{k}=0.5\left(\bar{\lambda}_{k}+\underline{\lambda}_{k}\right)$ for each $k$. In Table 2 , we summarize our numerical results for some randomly generated tensors. In this table, $n$ and $m$ specify the dimension and the order, respectively, of the randomly generated tensor. Ite denotes the number of iterations, $\bar{\lambda}-\underline{\lambda}$ and $\lambda$ denote the values of $\bar{\lambda}_{k}-\underline{\lambda}_{k}$ and $0.5\left(\bar{\lambda}_{k}+\underline{\lambda}_{k}\right)$, respectively, at the final iteration. $\left\|A x^{m-1}-\lambda x^{[m-1]}\right\|_{\infty}$ denotes the value of $\| A\left(x^{(k)}\right)^{m-1}-$ $\lambda_{k}\left(x^{(k)}\right)^{[m-1]} \|_{\infty}$ at the final iteration. The results reported in Table 2 show that the proposed method is promising. The algorithm is able to produce the largest eigenvalue for all these randomly generated tensors.

4. Higher-order Markov chains. In this section, we apply the method to compute the probability distribution of a higher-order Markov chain.

Data sequences (or time series) occur frequently in many real-world applications. The most important step in analyzing a data sequence (or time series) is the selection 
TABLE 1

Output of Algorithm 2.1 for Example 1.

\begin{tabular}{ccclcc}
\hline$k$ & $\underline{\lambda_{k}}$ & $\bar{\lambda}_{k}$ & $\bar{\lambda}_{k}-\underline{\lambda}_{k}$ & \multicolumn{1}{c}{$\lambda_{k}$} & $\left\|A\left(x^{k}\right)^{m-1}-\lambda_{k}\left(x^{k}\right)^{[m-1]}\right\|_{\infty}$ \\
\hline 1 & $4.18 \mathrm{e}+001$ & $4.48 \mathrm{e}+001$ & $3.07 \mathrm{e}+000$ & $4.33 \mathrm{e}+001$ & $5.78 \mathrm{e}-001$ \\
2 & $4.32 \mathrm{e}+001$ & $4.34 \mathrm{e}+001$ & $2.32 \mathrm{e}-001$ & $4.33 \mathrm{e}+001$ & $4.50 \mathrm{e}-002$ \\
3 & $4.32 \mathrm{e}+001$ & $4.33 \mathrm{e}+001$ & $2.70 \mathrm{e}-002$ & $4.33 \mathrm{e}+001$ & $5.25 \mathrm{e}-003$ \\
4 & $4.33 \mathrm{e}+001$ & $4.33 \mathrm{e}+001$ & $2.80 \mathrm{e}-003$ & $4.33 \mathrm{e}+001$ & $5.46 \mathrm{e}-004$ \\
5 & $4.33 \mathrm{e}+001$ & $4.33 \mathrm{e}+001$ & $3.09 \mathrm{e}-004$ & $4.33 \mathrm{e}+001$ & $6.02 \mathrm{e}-005$ \\
6 & $4.33 \mathrm{e}+001$ & $4.33 \mathrm{e}+001$ & $3.35 \mathrm{e}-005$ & $4.33 \mathrm{e}+001$ & $7.12 \mathrm{e}-007$ \\
7 & $4.33 \mathrm{e}+001$ & $4.33 \mathrm{e}+001$ & $3.66 \mathrm{e}-006$ & $4.33 \mathrm{e}+001$ & $7.75 \mathrm{e}-008$ \\
8 & $4.33 \mathrm{e}+001$ & $4.33 \mathrm{e}+001$ & $3.98 \mathrm{e}-007$ & $4.33 \mathrm{e}+001$ & $8.44 \mathrm{e}-009$ \\
9 & $4.33 \mathrm{e}+001$ & $4.33 \mathrm{e}+001$ & $4.33 \mathrm{e}-008$ & $4.33 \mathrm{e}+001$ & \\
\hline
\end{tabular}

TABLE 2

Numerical results of Algorithm 2.1 for randomly generated tensors.

\begin{tabular}{lllc}
\hline$(n, m)$ & Ite & $\bar{\lambda}-\underline{\lambda}$ & $\left\|A x^{m-1}-\lambda x^{[m-1]}\right\|_{\infty}$ \\
\hline$(5,3)$ & 10 & $2.61 \mathrm{e}-008$ & $2.63 \mathrm{e}-009$ \\
$(5,4)$ & 7 & $5.56 \mathrm{e}-008$ & $2.56 \mathrm{e}-009$ \\
$(5,5)$ & 6 & $6.44 \mathrm{e}-009$ & $1.30 \mathrm{e}-010$ \\
$(5,6)$ & 7 & $6.37 \mathrm{e}-010$ & $5.74 \mathrm{e}-012$ \\
$(10,3)$ & 8 & $2.13 \mathrm{e}-008$ & $1.03 \mathrm{e}-009$ \\
$(10,4)$ & 7 & $1.93 \mathrm{e}-008$ & $3.08 \mathrm{e}-010$ \\
$(10,5)$ & 6 & $2.89 \mathrm{e}-008$ & $1.45 \mathrm{e}-010$ \\
$(10,6)$ & 6 & $5.82 \mathrm{e}-009$ & $9.32 \mathrm{e}-012$ \\
$(15,3)$ & 8 & $6.51 \mathrm{e}-009$ & $2.15 \mathrm{e}-010$ \\
$(15,4)$ & 7 & $6.13 \mathrm{e}-009$ & $5.25 \mathrm{e}-011$ \\
$(15,5)$ & 6 & $7.36 \mathrm{e}-009$ & $1.64 \mathrm{e}-011$ \\
$(20,3)$ & 8 & $3.11 \mathrm{e}-009$ & $8.08 \mathrm{e}-011$ \\
$(30,3)$ & 7 & $5.30 \mathrm{e}-008$ & $8.81 \mathrm{e}-010$ \\
$(40,3)$ & 7 & $7.82 \mathrm{e}-009$ & $9.94 \mathrm{e}-011$ \\
$(50,3)$ & 7 & $6.60 \mathrm{e}-009$ & $6.76 \mathrm{e}-011$ \\
$(60,3)$ & 7 & $2.48 \mathrm{e}-009$ & $2.05 \mathrm{e}-011$ \\
$(70,3)$ & 7 & $1.72 \mathrm{e}-009$ & $1.22 \mathrm{e}-011$ \\
$(80,3)$ & 7 & $1.87 \mathrm{e}-009$ & $1.19 \mathrm{e}-011$ \\
$(90,3)$ & 7 & $6.91 \mathrm{e}-010$ & $3.87 \mathrm{e}-012$ \\
$(100,3)$ & 7 & $1.05 \mathrm{e}-009$ & $5.23 \mathrm{e}-012$ \\
\hline
\end{tabular}

of an appropriate mathematical model for the data, because it helps in predictions, hypothesis testing, and rule discovery. A categorical data sequence $Z$ can be logically represented as a vector $\left(z_{1}, z_{2}, \ldots, z_{T}\right)$, where $T$ is the length of the sequence, and we consider that each data point $z_{t}$ in a data sequence takes values in $\{1,2, \ldots, n\}$ and $n$ is finite, i.e., has $m$ possible categories or states. For categorical data sequences, there are many situations in which one would like to employ higher-order Markov chain models as a mathematical tool, and a number of applications can be found in the literature; see, for instance, [5] and references therein. A higher-order Markov chain model is used to fit the observed data through the calculation of higher-order transition probabilities:

$$
0 \leq p_{k_{1}, k_{2}, \ldots, k_{m}}=\operatorname{Prob}\left(X_{t}=k_{1} \mid X_{t-1}=k_{2}, \ldots, X_{t-n}=k_{m}\right) \leq 1,
$$

where

$$
\sum_{k_{1}=1}^{n} p_{k_{1}, k_{2}, \ldots, k_{m}}=1
$$

To summarize, we can consider an $m$-order $n$-dimensional tensor $\mathcal{P}$ consisting of $n^{m}$ 
entries between 0 and 1 :

$$
\mathcal{P}=\left(p_{k_{1} k_{2} \ldots k_{m}}\right), \quad 1 \leq i_{1}, i_{2}, \ldots, i_{m} \leq n .
$$

In [5], several methods are employed to approximate higher-order Markov chains. For instance, the entry $p_{k_{1} k_{2} \ldots k_{m}}$ is approximated by a linear combination of $p_{k_{i}, k_{j}}$ for some $i$ and $j$, and therefore a higher-order Markov chain can be approximated by a linear combination of transition matrices. However, according to the results in Theorems 2.4 and 2.5, it is not necessary to approximate higher-order Markov chains. We have the following corollary about the probability distribution of a higher-order Markov chain.

COROLlaRY 4.1. If $\mathcal{P}$ is an irreducible nonnegative tensor of order $m$ and dimension $n$ with (4.2), then there exist $\lambda_{0}>0$ and a vector $\bar{x}>0, \bar{x} \in R^{n}$, such that

$$
\mathcal{P} \bar{x}^{m-1}=\lambda_{0} \bar{x}^{[m-1]} .
$$

In particular, we can normalize $\bar{x}$ such that

$$
\sum_{i=1}^{n} \bar{x}_{i}=1
$$

and therefore a probability distribution of a higher-order Markov chain can be defined. However, we may not call it a stationary probability distribution, as $\lambda_{0}$ may not equal 1; see the examples in the next subsection. This result is different from a second-order irreducible nonnegative tensor (a Markov chain matrix $P$ ) that the largest eigenvalue of a Markov chain matrix is always equal to one [1], and the corresponding eigenvector $x>0$; i.e., $P x=x$. Since the eigenvector $x$ can be normalized such that the sum of its entries is equal to one, it is also called a stationary probability distribution for $P$.

4.1. An application. In this subsection, we employ the iterative method to compute probability distributions of higher-order Markov chains. Such probability distributions can be used to characterize higher-order Markov chains for decision making. A large soft-drink company in Hong Kong presently faces an in-house problem of production planning and inventory control. A pressing issue that stands out is the storage space of its central warehouse, which often finds itself in the state of overflow or near capacity. The company thus urgently needs to study the interplay between its storage space requirements and its overall growing sales demand. There are various product states depending on the level of sales volume:

state 1: very slow-moving (very low sales volume),

state 2: slow-moving,

state 3: standard,

state 4: fast-moving,

state 5: very fast-moving (very high sales volume).

Such labellings are useful from both marketing and production planning points of view; see [5]. For instance, when we obtain the probability distribution of product states (states $1,2,3,4,5)$ by using Corollary 4.1 , then we can find the optimal policy for minimizing the average long-run cost of the production system for the products under these probabilities [4]. There are costs associated with each product remaining at the end of the day (overage cost) and each unsatisfied demand (shortage cost). This is a well-known classical problem in management science [8]. 
TABLE 3

Prediction accuracy $r$ in the sales demand data.

\begin{tabular}{|l|c|c|c|c|}
\hline & Product A & Product B & Product C & Product D \\
\hline $\begin{array}{l}\text { Second-order tensor } \\
\text { (Markov chain matrix) }\end{array}$ & 0.76 & 0.70 & 0.39 & 0.74 \\
\hline Third-order tensor & 0.79 & 0.78 & 0.51 & 0.83 \\
\hline Randomly chosen & 0.20 & 0.20 & 0.20 & 0.20 \\
\hline
\end{tabular}

Next we would like to make use of higher-order Markov models for the purpose of prediction. According to the state probability distribution, the prediction of the next state $\hat{z}_{t}$ at time $t$ can be taken as the state with the maximum probability, i.e.,

$$
\hat{z}_{t}=j \quad \text { if }\left[\hat{\mathbf{z}}_{t}\right]_{i} \leq\left[\hat{\mathbf{z}}_{t}\right]_{j} \forall 1 \leq i \leq n .
$$

To evaluate the performance and effectiveness of Markov chain models, a prediction result is measured by the prediction accuracy $r$, defined as

$$
r=\frac{\sum_{t=m+1}^{T} \delta_{t}}{T},
$$

where $T$ is the length of the data sequence and

$$
\delta_{t}= \begin{cases}1 & \text { if } \hat{z}_{t}=z_{t} \\ 0 & \text { otherwise }\end{cases}
$$

For our new model, we consider the third-order tensor model (i.e., $m=3$ ) and use the data to estimate the probabilities. The results are reported in Table 3. For comparison, we also study the second-order tensor (i.e., Markov chain matrix). Results show the effectiveness of the third-order tensor model. We see from Figure 1 that the change of the states of the products $\mathrm{A}, \mathrm{B}$, and $\mathrm{D}$ is more regular than that of the product C. In Table 4, we display the calculation results and the probability distributions computed by the iterative method. We see from the table that the computed values are not equal to 1 , and four different products have different probability distributions characterizing their corresponding higher-order Markov chains.

5. Conclusions. In this paper we have proposed an iterative method for calculating the largest eigenvalue of an irreducible nonnegative tensor. This method is an extension of a method of Collatz [6] for calculating the spectral radius of an irreducible nonnegative matrix. When the order of the nonnegative tensor is 2 , Theorem 2.4 reduces to the result by Collatz, and Algorithm 2.1 reduces to the Collatz algorithm $[6,13,14]$ for finding the spectral radius of an irreducible nonnegative matrix. In $[13,14]$, the following result was given.

TheOrem 5.1. Let $B$ be an irreducible nonnegative matrix. Assume that $\lambda_{0}$ is the unique positive eigenvalue of $B$ and that the two convergent sequences $\left\{\underline{\lambda}_{k}\right\}$ and $\left\{\bar{\lambda}_{k}\right\}$ are generated by the Collatz algorithm. Let $\underline{\lambda}=\lim _{k \rightarrow+\infty} \underline{\lambda}_{k}$ and $\bar{\lambda}=\lim _{k \rightarrow+\infty} \bar{\lambda}_{k}$. Then, $\lambda_{0}=\underline{\lambda}=\bar{\lambda}$ if and only if $B$ is primitive.

In a recent paper by Chang, Pearson, and Zhang [2], some conditions have been given for the positive eigenvalue of an irreducible nonnegative tensor to be real geometrically simple. We conjecture that in Theorem 2.5 if the positive eigenvalue $\lambda_{0}$ of the irreducible nonnegative tensor $\mathcal{A}$ is real geometrically simple, then $\lambda_{0}=\underline{\lambda}=\bar{\lambda}$ for $m>2$. While we have enough numerical evidence as well as intuition to believe that this is true, we do not yet have a proof, so we leave it as an open problem. We note 

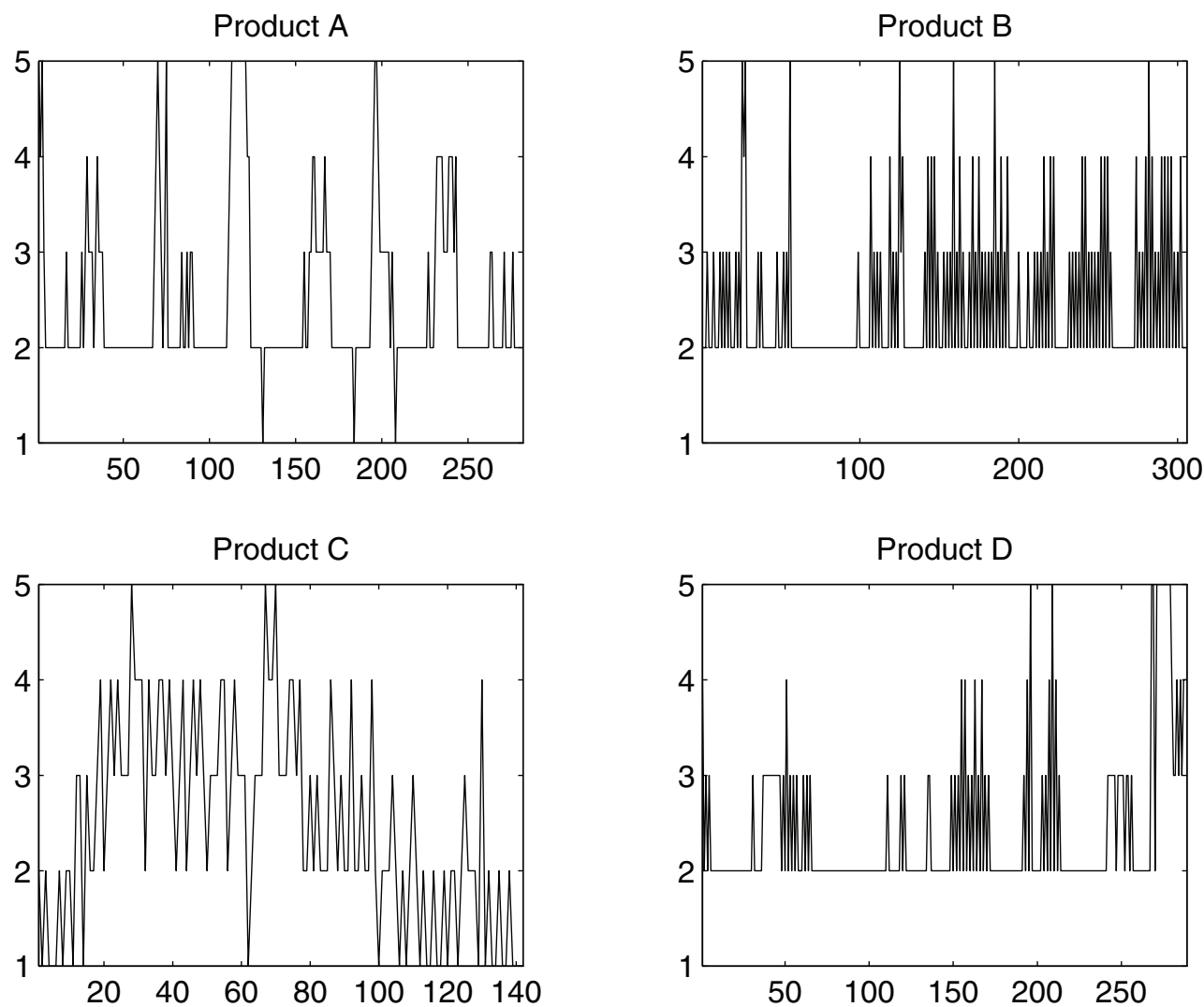

FIG. 1. The states of four products $A, B, C$, and $D$.

TABLE 4

Computed results by the iterative method.

\begin{tabular}{|l|c|c|c|c|}
\hline & Product A & Product B & Product C & Product D \\
\hline$\lambda$ & 4.2900 & 4.6171 & 4.5247 & 4.7198 \\
\hline Probability & 0.1139 & 0.1364 & 0.1918 & 0.1214 \\
distribution & 0.3509 & 0.3062 & 0.2687 & 0.2568 \\
for five & 0.2089 & 0.1985 & 0.2603 & 0.2416 \\
states & 0.1714 & 0.1939 & 0.1910 & 0.2064 \\
& 0.1550 & 0.1650 & 0.0882 & 0.1737 \\
\hline
\end{tabular}

that the conjecture is false for $m=2$. In particular, a matrix may be nonnegative and irreducible with simple eigenvalues but not primitive, for example,

$$
\left(\begin{array}{lll}
0 & 1 & 1 \\
1 & 0 & 0 \\
1 & 0 & 0
\end{array}\right) .
$$

Acknowledgments. The authors would like to thank the reviewers for their suggestions to improve the presentation of the paper. In particular, one reviewer raised that the conjecture is false for $m=2$, and provided an example. 


\section{REFERENCES}

[1] A. Berman and R. J. Plemmons, Nonnegative Matrices in the Mathematical Sciences, Classics in Appl. Math. 9, SIAM, Philadelphia, 1994.

[2] K. C. Chang, K. Pearson and T. Zhang, Perron-Frobenius theorem for nonnegative tensors, Comm. Math. Sci., 6 (2008), pp. 507-520.

[3] K. C. Chang, K. Pearson and T. Zhang, On eigenvalue problems of real symmetric tensors, J. Math. Anal. Appl., 350 (2009), pp. 416-422.

[4] W. Ching, E. Fung, And M. NG, Higher-order Markov model for the Newsboy's problem, J. Oper. Res. Soc., 54 (2003), pp. 291-298.

[5] W. Ching and M. NG, Markov Chains: Models, Algorithms and Applications, Int. Ser. Oper. Res. Manag. Sci., Springer, New York, 2006.

[6] L. Collatz, Einschliessungssatz für die charakteristischen Zahlen von Matrizen, Math. Zeit., 48 (1942), pp. 221-226.

[7] L.-H. Lim, Singular values and eigenvalues of tensors: A variational approach, in Proceedings of the IEEE International Workshop on Computational Advances in Multi-Sensor Adaptive Processing (CAMSAP '05), Vol. 1, IEEE Computer Society Press, Piscataway, NJ, 2005, pp. 129-132.

[8] S. Nahmias, Production and Operation Analysis, McGraw-Hill, Chicago, 1997.

[9] L. QI, Eigenvalues of a real supersymmetric tensor, J. Symbolic Comput., 40 (2005), pp. 13021324.

[10] L. QI, Eigenvalues and invariants of tensor, J. Math. Anal. Appl., 325 (2007), pp. 1363-1377.

[11] L. QI, W. Sun, AND Y. WANG, Numerical multilinear algebra and its applications, Frontiers Math. China, 2 (2007), pp. 501-526.

[12] L. QI, Y. WANG, AND E.X. WU, D-eigenvalues of diffusion kurtosis tensor, J. Comput. Appl. Math., 221 (2008), pp. 150-157.

[13] R. VArGa, Matrix Iterative Analysis, Prentice-Hall, Englewood Cliffs, NJ, 1962.

[14] R. J. Wood ANd M. J. O'NeILL, Finding the spectral radius of a large sparse nonnegative matrix, ANZIAM J., 48 (2007), pp. C330-C345.

Copyright (c) by SIAM. Unauthorized reproduction of this article is prohibited. 